\title{
An Ocean Wind Doppler Model Based on the Generalized Curvature Ocean Surface Scattering Model
}

\author{
Said Faozi ${ }^{1,{ }^{*}}$, Johnsen Harald ${ }^{2,{ }^{*}}$, Chapron Bertrand ${ }^{3,{ }^{*}}$, Engen Geir ${ }^{2,{ }^{*}}$
}

${ }^{1}$ NOAA, Ocean Surface Winds Team, Ctr Satellite Applicat \& Res, College Pk, MD 20740 USA.

${ }^{2}$ Norut, Earth Observat Grp, N-9294 Tromso, Norway.

${ }^{3}$ IFREMER, Lab Oceanog Space, F-29280 Brest, France.

* Corresponding authors : email addresses : faozi.said@noaa.gov ; harald.johnsen@norut.no ; bertrand.chapron@ifremer.fr ; geir.engen@norut.no

\begin{abstract}
:
A Doppler centroid D-C model based on the generalized curvature ocean surface scattering model (generalized curvature model or GCM) is presented. Two key features are included in this model: a skewness-related phase coefficient based on empirical skewness coefficients of sea-surface-slope probability density function (pdf) for wind speed less than $10 \mathrm{~m} / \mathrm{s}$ and effects from wave breaking for wind speed greater than $10 \mathrm{~m} / \mathrm{s}$. Simulated D-c values are exclusively compared with the empirical geophysical Doppler model function named CDOP, for hh and vv polarizations, various wind conditions, and incidence angles. Good agreement is found overall between CDOP and simulated D-C values. The overall bias for simulated Dc-vv with and without skewness are 2.63 versus $-0.51 \mathrm{~Hz}(14.6$ versus -2.8 $\mathrm{cm} / \mathrm{s})$, respectively; overall standard deviations are 2.76 versus $3.53 \mathrm{~Hz}(15.3$ versus $19.6 \mathrm{~cm} / \mathrm{s})$. For simulated DC-hh, overall bias values with and without skewness are -0.16 versus $-2.52 \mathrm{~Hz}(-0.9$ versus $-14 \mathrm{~cm} / \mathrm{s})$; standard deviations are 3.56 versus $4.32 \mathrm{~Hz}(19.7$ versus $24 \mathrm{~cm} / \mathrm{s})$. The overall bias for simulated Dc-vv with and without the wave breaking component are -0.08 versus $0.12 \mathrm{~Hz}(-0.4$ versus $0.7 \mathrm{~cm} / \mathrm{s}$ ), respectively; corresponding standard deviations are 3.32 versus $4.75 \mathrm{~Hz}$ (18.4 versus 26.3 $\mathrm{cm} / \mathrm{s}$ ). Bias values for simulated Dc-vv with and without the wave breaking component are -1.83 versus $-2.02 \mathrm{~Hz}(-10.2$ versus $-11.2 \mathrm{~cm} / \mathrm{s})$, with corresponding overall standard deviations of 3.43 versus 4.87 $\mathrm{Hz}(19$ versus $27 \mathrm{~cm} / \mathrm{s})$. The largest deviation from CDOP, of about $18 \mathrm{~Hz}(0.99 \mathrm{~m} / \mathrm{s})$, is found in the upwind direction for a 26 incidence angle, $10-\mathrm{m} / \mathrm{s}$ wind speed, and hh polarization.
\end{abstract}

Keywords : Doppler measurements, geophysical measurement techniques, sea surface, synthetic aperture radar (SAR) 


\section{INTRODUCTION}

To this date, very few known Doppler models are capable of relating the Doppler centroid $\left(D_{c}\right)$ to the sea surface wind field. Johannessen et al. [1] provides a Doppler model named DopRim to estimate ocean mesoscale dynamics such as ocean currents. This model uses the straightforward definition of the Doppler centroid $f_{D}$ provided by Chapron et al. [2] where

$$
f_{D}=\frac{k_{r}}{\pi} \frac{\left\langle\left(u \sin \theta_{i}-w \cos \theta_{i}\right) \sigma^{0}\left(\theta_{i}+\Delta \theta_{i}\right)\right\rangle}{\left\langle\sigma^{0}\left(\theta_{i}+\Delta \theta_{i}\right)\right\rangle} .
$$

In this equation, $k_{r}$ represents the radar wave number, $u$ and $v$ represent the magnitudes of the horizontal and vertical velocities, $\sigma^{0}$ represents the normalized radar cross section, $\theta_{i}$ is the incidence angle, and $\Delta \theta_{i}$ represents the effect on $\theta_{i}$ due to the local tilt induced by the longer waves. As shown in (1), the $D_{c}$ is dependent on $\sigma^{0}$ measurements requiring a backscatter model; a two-scale asymptotic decomposition is selected as part of the DopRim, which includes hydrodynamic and tilt modulations of the scattering facets (see [1] for more details). A second Doppler model known in the litterature is the model provided by Romeiser and Thompson, which appears to provide similar performance to the DopRim when Bragg scattering dominates [3].

Mouche et al. [4] proposes a semi-empirical Doppler model function using collocated ECMWF data with $v v$ and $h h$ Doppler centroid measurements from ASAR WSM products. A look-up table is then built using a three-layer neural network which is now known as CDOP with

$$
D_{c}=C_{D O P}\left(\phi, u_{10}, \theta_{i}, p o l\right)
$$

where $\phi$ is the relative wind direction, $u_{10}$ is the wind speed at $10 \mathrm{~m}$ height, $\theta_{i}$ represents the incidence angle, and pol the polarization state. Using the CDOP GMF from [4], Fig. 1 shows the $D_{c}$ in terms of the wind direction for a $10 \mathrm{~m} / \mathrm{s}$ wind speed and $v v$ polarization, given three different incidence angles. Four distinctive $D_{c}$ features are seen from this figure:

- the $D_{c}$ extrema occur in both up- and down-wind

- the extremum always occurs in up-wind regardless of the wind conditions and incidence angle

- the absolute $D_{c}$ decreases as $\theta_{i}$ increases for a given polarization, wind speed, and direction

- between up- and down-wind, the $D_{c}$ varies monotonously regardless of the incidence angle.

[4]-[6] show the advantage of combining both $\sigma^{o}$ and $D_{c}$ measurements from SAR data for sea surface wind retrieval purposes, where the inclusion of the $D_{c}$ in the wind inversion helps 
narrowing down the number of wind ambiguities. Whereas [4] uses CDOP as a valid Doppler model function, [6] uses a theoretical GMF based on the generalized curvature ocean surface scattering model [generalized curvature model (GCM)] [7]. The latter shares some similarities with [1] where a model for the NRCS is also required. Such a model has been developed where both small perturbation method (SPM) [8], the Kirchhoff approximation (KA) [9] results, as well as fundamental laws of reciprocity and tilt invariance are preserved up to first order [7]. In the following section, a description of the Doppler centroid derivation based on the GCM is provided, including necessary key improvements such as a skewness phase related coefficient and effects from wave breaking. Simulated $D_{c}$ are then compared against CDOP for various wind conditions, incidence angles, and polarization.

\section{DOPPLER CENTROID MODEL DESCRIPTION}

In this section, we provide a high level description of the Doppler centroid model derivation based on the work from [10]. This model is based on the assumption that the $D_{c}$ can be found by computing the zeroth and first moments of the power spectral density (PSD) of the complex SAR image: the zeroth moment represents $\sigma^{o}$ while the first moment normalized to the zeroth moment corresponds to the $D_{c}$.

\section{A. Basic derivation}

The complex SAR raw-data image can be found by first simulating the convolution of the transmitted SAR signal with the ocean surface reflectivity such that

$$
I_{\text {raw }}(\mathbf{x})=\int d \mathbf{l} e^{-\imath \mathbf{Q}_{h} \cdot \mathbf{l}} \gamma(\mathbf{l}, t) S(\mathbf{x}-\mathbf{l} ; R),
$$

where $S$ is the SAR signal spreading function, $\gamma$ is the ocean surface reflectivity, $\mathbf{x}$ is in groundrange coordinates, $R$ is the slant-range distance of the imaged object, and $t$ is the time; $\mathbf{Q}_{h}=$ $\mathbf{k}_{h}^{s}-\mathbf{k}_{h}^{i}$ is the component of the Ewald vector $\mathbf{Q}_{r}$ where $\mathbf{k}_{h}$ is the horizontal projection of the radar wave vector $\mathbf{k}_{r}=\left(\mathbf{k}_{h}, k_{z}\right)$, and the superscripts $i$ and $s$ refers to incident and scattered, respectively. The ocean surface reflectivity $\gamma$ can be expressed as

$$
\gamma\left(\mathbf{x}_{o}, t\right)=\int d \mathbf{x} \delta\left(\mathbf{x}+\mathbf{k}_{h} \frac{k_{z}}{k_{h}^{2}} \eta(\mathbf{x}, t)-\mathbf{x}_{o}\right) F(\mathbf{x}, t),
$$


where $\eta$ is the sea surface elevation and $F$ is a source function caused by the electric surface current defined up to first order such that

$$
F(\mathbf{x})=F^{(0)}+F^{(1)}(\mathbf{x})
$$

with

$$
F^{(0)}=\hat{\mathbf{H}}_{s} \cdot\left\{\mathcal{B}_{v v}^{(0)} \hat{\mathbf{v}}^{(0)} \hat{\mathbf{v}}^{(0)}+\mathcal{B}_{h h}^{(0)} \hat{\mathbf{h}}^{(0)} \hat{\mathbf{h}}^{(0)}\right\} \cdot \hat{\mathbf{H}}_{i},
$$

and

$$
F^{(1)}(\mathbf{x})=\int d \mathbf{k} e^{\imath \mathbf{k} \cdot \mathbf{x}} T_{F^{(1)}}(\mathbf{k}) \hat{\eta}(\mathbf{k})
$$

where

$$
T_{F^{(1)}}(\mathbf{k})=\imath Q_{z} F^{(0)}+\hat{\mathbf{H}}_{s} \cdot\left\{\mathcal{B}_{v v}^{(1)} \hat{\mathbf{v}}^{(1)} \hat{\mathbf{v}}^{(1)}+\mathcal{B}_{h h}^{(1)} \hat{\mathbf{h}}^{(1)} \hat{\mathbf{h}}^{(1)}\right\} \cdot \hat{\mathbf{H}}_{i}
$$

Further details about the variables in (6) through (8) can be found in both [11] and [7].

The complex SAR image spectrum can be found by applying both the principle of stationary phase and a matched filter to (3). The resulting compressed complex SAR image spectrum is

$$
\hat{I}_{c}(\mathbf{k}) \approx \int d \mathbf{x} e^{-\imath\left(\mathbf{Q}_{h} \cdot \mathbf{x}+Q_{z} \eta\right)} F\left(\mathbf{x}, t_{s}\right) U\left(x_{s}\right) V\left(y_{s}\right)
$$

where $U$ and $V$ are radar beam pattern variables directly related to the signal spreading function $S$, and $x_{s}, y_{s}$, and $t_{s}$ correspond to the stationary phase values (for more details about these quantities and the derivation of (9), see [10]).

As previously mentioned, the zeroth and first moments of the PSD of the compressed SAR signal are necessary to estimate the $D_{c}$. Using the result from (9), they are expressed as

$$
m_{0}=\int d \mathbf{k}\left\langle\hat{I}_{c}(\mathbf{k}) \hat{I}_{c}^{*}(\mathbf{k})\right\rangle
$$

and

$$
m_{1}=\int d \mathbf{k} k_{y}\left\langle\hat{I}_{c}(\mathbf{k}) \hat{I}_{c}^{*}(\mathbf{k})\right\rangle
$$

The Doppler centroid (in unit of Hertz) in terms of the first two spectral moments is

$$
D_{c}=\frac{1}{2 \pi} \frac{m_{1}}{m_{0}}
$$

which leads to

$$
D_{c}=\frac{1}{2 \pi} \frac{\int d \mathbf{x} e^{-\imath \mathbf{Q}_{h} \cdot \mathbf{x}}\left\langle e^{\imath(\zeta(\mathbf{0})-\zeta(\mathbf{x}))} f(\mathbf{x}) f^{*}(\mathbf{0})(\dot{\zeta}(\mathbf{x})+\dot{\zeta}(\mathbf{0}))\right\rangle}{\int d \mathbf{x} e^{-\imath \mathbf{Q}_{h} \cdot \mathbf{x}}\left\langle e^{\imath(\zeta(\mathbf{0})-\zeta(\mathbf{x}))} \Upsilon(\mathbf{x}, \mathbf{0})\right\rangle}
$$


In the above equation we set

$$
\zeta(\mathbf{x})=Q_{z} \eta(\mathbf{x})
$$

and

$$
f(\mathbf{x})=\underbrace{F^{(0)}}_{f^{(0)}}+\underbrace{F^{(1)}(\mathbf{x})}_{f^{(1)}} .
$$

Assuming the variables present in both Fourier kernels in (13) are Gaussian, and using properties of multivariate normal characteristic functions, we can now write

$$
\begin{aligned}
\begin{aligned}
\left.\imath e^{\imath(\zeta(\mathbf{0})-\zeta(\mathbf{x}))} f(\mathbf{x}) f^{*}(\mathbf{0})\right\rangle & =e^{\varphi_{\zeta \zeta}(\mathbf{x})-\varphi_{\zeta \zeta}(\mathbf{0})} \\
& {\left[\varphi_{f^{(1)} f^{(1)}}(\mathbf{x})+\left(f^{(0)}+\imath\left(\varphi_{f^{(1)} \zeta}(\mathbf{x})-\varphi_{f^{(1)} \zeta}(\mathbf{0})\right)\right)\right.} \\
& \left.\left(f^{*(\mathbf{0})}-\imath\left(\varphi_{\zeta f^{(1)}}(\mathbf{x})-\varphi_{\zeta f^{(1)}}(\mathbf{0})\right)\right)\right]
\end{aligned}
\end{aligned}
$$

and

$$
\begin{aligned}
& \left\langle e^{\imath(\zeta(\mathbf{0})-\zeta(\mathbf{x}))} f(\mathbf{x}) f^{*}(\mathbf{0})(\dot{\zeta}(\mathbf{x})+\dot{\zeta}(\mathbf{0}))\right\rangle=e^{\varphi_{\zeta \zeta}(\mathbf{x})-\varphi_{\zeta \zeta}(\mathbf{0})} \\
& {\left[\imath f^{(0)} \varphi_{\dot{\zeta} \zeta}(\mathbf{x})\left(f^{*(\mathbf{0})}-\imath\left(\varphi_{\zeta f^{(1)}}(\mathbf{x})-\varphi_{\zeta f^{(1)}}(\mathbf{0})\right)\right)\right.} \\
& -\imath f^{*(0)} \varphi_{\zeta \dot{\zeta}}(\mathbf{x})\left(f^{(\mathbf{0})}+\imath\left(\varphi_{f^{(1)} \zeta}(\mathbf{x})-\varphi_{f^{(1)} \zeta}(\mathbf{0})\right)\right) \\
& -f^{(0)} \varphi_{\zeta \dot{\zeta}}(\mathbf{x})\left(\varphi_{\zeta f^{(1)}}(\mathbf{x})-\varphi_{\zeta f^{(1)}}(\mathbf{0})\right) \\
& -f^{*(0)} \varphi_{\dot{\zeta} \zeta}(\mathbf{x})\left(\varphi_{f^{(1)} \zeta}(\mathbf{x})-\varphi_{f^{(1)} \zeta}(\mathbf{0})\right) \\
& +f^{*(0)}\left(\varphi_{f^{(1)} \dot{\zeta}}(\mathbf{x})+\varphi_{f^{(1)} \dot{\zeta}}(\mathbf{0})\right) \\
& +f^{(0)}\left(\varphi_{\dot{\zeta} f^{(1)}}(\mathbf{x})+\varphi_{\dot{\zeta} f^{(1)}}(\mathbf{0})\right) \\
& -\imath\left(\varphi_{f^{(1)} \dot{\zeta}}(\mathbf{x})+\varphi_{f^{(1)} \dot{\zeta}}(\mathbf{x})\right)\left(\varphi_{\zeta f^{(1)}}(\mathbf{x})-\varphi_{\zeta f^{(1)}}(\mathbf{0})\right) \\
& +\imath\left(\varphi_{\dot{\zeta} f^{(1)}}(\mathbf{x})+\varphi_{\dot{\zeta} f^{(1)}}(\mathbf{x})\right)\left(\varphi_{f^{(1)} \zeta}(\mathbf{x})-\varphi_{f^{(1)} \zeta}(\mathbf{0})\right) \\
& +\imath \varphi_{f^{(1)} f^{(1)}}(\mathbf{x})\left(\varphi_{\dot{\zeta} \zeta}(\mathbf{x})-\varphi_{\zeta \dot{\zeta}}(\mathbf{x})\right) \\
& +\imath\left(\varphi_{\dot{\zeta} \zeta}(\mathbf{x})-\varphi_{\zeta \dot{\zeta}}(\mathbf{x})\right)\left(\varphi_{f^{(1)} \zeta}(\mathbf{x})-\varphi_{f^{(1)} \zeta}(\mathbf{0})\right) \\
& \left.\left(\varphi_{\zeta f^{(1)}}(\mathbf{x})-\varphi_{\zeta f^{(1)}}(\mathbf{0})\right)\right] .
\end{aligned}
$$

Following the same procedure from [11], the various covariance functions $\varphi_{a b}$ present in (16) and (17) can be computed using the relation

$$
\varphi_{a b}(\mathbf{x}, t)=\operatorname{Re}\left\{\frac{1}{(2 \pi)^{2}} \int d \mathbf{k} e^{\imath(\mathbf{k} \cdot \mathbf{x}-w t)} T_{a}(\mathbf{k}) T_{b}{ }^{*}(\mathbf{k}) \Gamma(\mathbf{k})\right\},
$$


where $T_{a}(\mathbf{k})$ and $T_{b}(\mathbf{k})$ are transfer functions, and $\Gamma(\mathbf{k})$ is the power spectral density of the sea surface elevation [12]. The required transfer functions are

$$
\begin{gathered}
T_{f^{(1)}}(\mathbf{k})=T_{F^{(1)}}(\mathbf{k}), \\
T_{\zeta}(\mathbf{k})=Q_{z}, \\
T_{\dot{\zeta}}(\mathbf{k})=-\imath(g k)^{1 / 2} T_{\zeta}(\mathbf{k}),
\end{gathered}
$$

where $g$ is the acceleration of gravity.

\section{B. Lagrangian implementation with a skewness related phase coefficient}

We follow the same method described in [11] for the $D_{c}$ simulation where the horizontal displacement of the fluid particles is followed such that

$$
\tilde{\mathbf{x}}=\mathbf{x}+\boldsymbol{\xi}(\mathbf{x}, \alpha),
$$

where $\boldsymbol{\xi}(\mathbf{x}, \alpha)$ refers to the horizontal displacement of a fluid particle on the sea surface at a reference point $\mathrm{x}$, related to the orbital motion of the particle being described as $\chi(\mathbf{x})=$ $(\boldsymbol{\xi}(\mathbf{x}), \eta(\mathbf{x})) ; \alpha$ represents a phase coefficient which introduces an asymmetry to the vertical wave profile. In order to determine its value, we first generate several 3D sea surfaces for various values of $\alpha$ and $u 10$, and compute the gradient of the generated surfaces in order to find the skewness coefficient of the sea surface slope. A best fit is then performed with the empirical up/down-wind skewness coefficient $c_{03}$ of the sea surface slope pdf derived by both Cox \& Munk [13] and Bréont \& Henriot [14] respective experiments. As shown in [11], we find the following relationship

$$
\alpha\left(\mathrm{u}_{10}\right) \approx 1.75 c_{03} .
$$

The change of variable from (22) alters the transfer functions in (19) and (20)

$$
T_{f^{(1)}}(\mathbf{k})=-k F^{(0)} e^{i \alpha}+T_{F^{(1)}}(\mathbf{k}),
$$

and

$$
T_{\zeta}(\mathbf{k})=\imath \mathbf{Q}_{h} \cdot \hat{\mathbf{k}} e^{i \alpha}+Q_{z} .
$$




\section{Wave breaking $D_{c}$ component}

Similar to [15], a wave breaking component is also implemented for the GCM based $D_{c}$ model, which enables $D_{c}$ estimation from surface scattering, specular reflection, and wavebreaking sources. The theory is based on the work of Kudryavtsev et al. [16], where applied to the Doppler centroid yields

$$
D_{c-t o t}^{\alpha \alpha}=P_{g c m}^{\alpha \alpha} D_{c-g c m}^{\alpha \alpha}(1-q)+P_{w b}^{\alpha \alpha} D_{c-w b} q
$$

where $D_{c-t o t}^{\alpha \alpha}$ represents the total geophysical Doppler from wind generated waves, $D_{c-g c m}^{\alpha \alpha}$ represents the Doppler contribution obtained from the GCM including both Bragg and specular scattering, $D_{c-w b}$ is the Doppler contribution from wave breaking, and the superscript $\alpha \alpha$ refers to the polarization state (i.e. $\alpha \alpha \in\{h h, v v\}$ ); the $q$ factor is based on [16] and is defined such that

$$
q=\frac{c_{q}}{2 \alpha_{g}} \int_{\phi} d \phi\left[\int_{k<10 k_{p}} \beta(k, \phi) B_{p}(k, \phi) d \ln k+\int_{10 k_{p}}^{k_{n b}} \beta(k, \phi) B_{e q 0}(k, \phi) d \ln k\right],
$$

where $c_{q}=10.5, \alpha_{g}=5 \cdot 10^{-3}, k_{p}$ is the peak wave number, $k_{n b}=\min \left(k_{r} / 10 \mathrm{~m}, 2 \pi / 0.3 \mathrm{~m}\right)$, $\beta(k, \phi)$ is the wind growth rate as suggested from [16], $B_{p}(k, \phi)$ is the ocean curvature spectrum directly related to $\Gamma(\mathbf{k})$ in (18), and $B_{e q 0}(k, \phi)$ is the reference equilibrium spectrum. Finally, the coefficient $P_{X X}^{\alpha \alpha}$ is

$$
P_{X X}^{\alpha \alpha}=\frac{\sigma_{X X}^{o}}{\sigma^{o}}
$$

where the subscript $X X$ refers to either $g \mathrm{~cm}$ or $w b$, and

$$
\sigma^{o}=\sigma_{g c m}^{o}(1-q)+\sigma_{w b}^{o} q
$$

as defined in [15].

The wave breaking component in (26) [i.e. $D_{c-w b}$ ] is based on (6) from [1] where

$$
D_{c-w b}=-\frac{\sin \theta_{i} k_{r}}{\pi} \frac{q^{\prime}}{q}
$$

where $q^{\prime}$ has the same expression as $q$ except with the inclusion of the phase velocity in both integrands of (27). 


\section{Simulation ANALYSis}

The GCM based $D_{c}$ model is now compared with and without the skewness related phase parameter (based on Cox \& Munk skewness coefficient) against CDOP for both $h h$ and $v v$ polarizations, for low to moderate wind speeds and various incidence angles. Figure 2 shows this comparison for $\mathrm{u}_{10}=5,7$ and $10 \mathrm{~m} / \mathrm{s}$ and all wind directions where we now note an asymmetry in the up/down-wind direction when skewness is included. Table I also provides corresponding statistical results such as bias and standard deviation values of estimated $D_{c}$ values with and without skewness against CDOP. We note a definite improvement overall in the $D_{c}$ estimation when the skewness component is included. For the $h h$ polarization, the overall bias (for all incidence angles and wind speeds) for simulated $D_{c}$ with and without skewness are 2.63 vs. $-0.51 \mathrm{~Hz}(14.6$ vs. $-2.8 \mathrm{~cm} / \mathrm{s})$, respectively; overall standard deviations are 2.76 vs. $3.53 \mathrm{~Hz}(15.3$ vs. $19.6 \mathrm{~cm} / \mathrm{s})$. For the $v v$ polarization, overall bias values for simulated $D_{c}$ with and without skewness are -0.16 vs. $-2.52 \mathrm{~Hz}(-0.9$ vs. $-14 \mathrm{~cm} / \mathrm{s})$, respectively; overall standard deviations are 3.56 vs. $4.32 \mathrm{~Hz}$ (19.7 vs. $24 \mathrm{~cm} / \mathrm{s})$.

For $\mathrm{u}_{10}=5 \mathrm{~m} / \mathrm{s}$, table I shows improved bias values when the skewness component is implemented for the $v v$ polarization and for all incidence angles. For the $h h$ polarization, the bias values are similar to those of the $D_{c}$ without skewness, but with improved standard deviation values.

When $\mathrm{u}_{10}=7 \mathrm{~m} / \mathrm{s}$, the $D_{c-h h}$ with the skewness component is overestimated as the wind speed increases in the up/down-wind direction for incidence angle below 40 degrees as shown in Fig. 2. This observation is also supported by the larger bias values compared to the 'no skewness case' found in Table I. We note improved results for the $D_{c-v v}$ with the skewness component, except that it is particularly underestimated around the up-wind direction for incidence angle values around 40 degrees.

For $\mathrm{u}_{10}=10 \mathrm{~m} / \mathrm{s}$, the $D_{c-h h}$ with the skewness component is overestimated around the upwind direction for all incidence angles. Its performance is only superior to the 'no skewness case' for high incidence angle as shown in Table I. The $D_{c-v v}$ with the skewness component is also overestimated in the up-wind direction for low to mid incidence angles. For incidence angle around 40 degrees, improved results over the 'no skewness case' are obtained for the $v v$ polarization as shown in Table I. 
For wind speeds greater than $10 \mathrm{~m} / \mathrm{s}$, the wave breaking component described in subsection II-C is included in the $D_{c}$ simulation with the skewness component omitted. This is due to the fact that empirical skewness coefficients of pdf of slope from Cox \& Munk have been partly used for the derivation of the wave breaking component (see [16] for details). Table II shows improved statistical results, compared the 'no skewness case', across all given incidence angles and wind speeds. For the $h$ polarization, the mean bias (for all incidence angles and wind speeds) for simulated $D_{c}$ with and without the wave breaking component are -0.08 vs. $0.12 \mathrm{~Hz}(-0.4$ vs. 0.7 $\mathrm{cm} / \mathrm{s})$, respectively; mean standard deviations are 3.32 vs. $4.75 \mathrm{~Hz}(18.4$ vs. $26.3 \mathrm{~cm} / \mathrm{s})$. For the $v v$ polarization, mean bias values for simulated $D_{c}$ with and without wave breaking effects are -1.83 vs. $-2.02 \mathrm{~Hz}(-10.2$ vs. $-11.2 \mathrm{~cm} / \mathrm{s})$, respectively; corresponding mean standard deviations are 3.43 vs. $4.87 \mathrm{~Hz}\left(19\right.$ vs. $27 \mathrm{~cm} / \mathrm{s}$ ). Figure 3 shows that the $D_{c-h h}$ with wave breaking provides a good match with CDOP for incidence angle equal or greater than 32 degrees for both $\mathrm{u}_{10}=10$ and $12 \mathrm{~m} / \mathrm{s}$; whereas for lower incidence angle, both $D_{c-h h}$ and $D_{c-v v}$ noticeably differ in the up/down-wind direction from CDOP. For incidence angle around 40 degrees, simulated $D_{c-v v}$ with wave breaking provides improved results but are still underestimated around the up-wind direction compared to CDOP. In fact, the latest version of the DopRim model [17] also includes breaking wave effects using a similar approach as presented in this paper. Although the GCMDop and DopRim differ, we see some similar trends such as underestimation of Doppler in the up-wind direction at high incidence angle and higher wind speed (see [17]). We also observe that both models perform best at low wind speeds $(5 \mathrm{~m} / \mathrm{s})$ and moderate incidence angles $(32$ deg) for both polarizations.

\section{CONClusion}

An ocean wind Doppler centroid model based on the generalized curvature model is presented. Two key features are included in this $D_{c}$ model, namely a skewness phase related coefficient and effects from wave breaking. Simulated $D_{c}$ are then compared with CDOP for both $h h$ and $v v$ polarizations, including various wind conditions and incidence angles. Overall, simulated $D_{c}$ values offer improved results and are in good agreement with CDOP. An up/down-wind asymmetry is now present when including either the skewness phase related coefficient or the wave breaking component. We note however an overestimation of the $D_{c}$ particularly in the up-wind direction for wind speeds between 7 and $10 \mathrm{~m} / \mathrm{s}$, incidence angles less than 32 degrees, 
and for both polarizations. Although the $D_{c}$ with either the skewness or wave breaking term provides improved results over the 'no skewness/wave breaking' case, underestimation of around $5 \mathrm{~Hz}(27.7 \mathrm{~cm} / \mathrm{s})$ is still present again around the up-wind direction for the $v v$ polarization and a 40 degree incidence angle compared to CDOP.

With the recent launch of the Sentinel-1 instrument, future works could certainly include a validation of the GCM based $D_{c}$ model by performing ocean wind retrievals using the Sentinel $D_{c}$ measurements; as presented in [6], ocean wind retrievals can be done when combining the $D_{c}$ with NRCS measurements from SAR stripmap data. Results could then be validated against ground truth data, and compared with similar experiments using either CDOP or the DopRim model.

\section{ACKNOWLEDGMENT}

Funding for this work has been provided by the Troms County Regional Development fund (RDA).

\section{REFERENCES}

[1] J. A. Johannessen, B. Chapron, F. Collard, V. Kudryavtsev, A. Mouche, D. Akimov, and K.-F. Dagestad, "Direct ocean surface velocity measurements from space: Improved quantitative interpretation of Envisat ASAR observations," Geophys. Res. Lett., vol. 35, November 2008.

[2] B. Chapron, F. Collard, and F. Ardhuin, "Direct measurements of ocean surface velocity from space: interpretation and validation,” Journal of Geophysical Research, vol. 110, no. C07008, 2005, doi:10.1029/2004JC002809.

[3] R. Romeiser and D. R. Thompson, "Numerical study on the along-track interferometric radar imaging mechanism of oceanic surface currents," IEEE Transactions on Geoscience and Remote Sensing, vol. 38, no. 1, pp. 446-458, 2000.

[4] A. A. Mouche, F. Collard, B. Chapron, K.-F. Dagestad, G. Guitton, J. A. Johannessen, V. Kerbaol, and M. W. Hansen, "On the use of Doppler shift for sea surface wind retrieval from SAR," Geoscience and Remote Sensing, IEEE Transactions on, vol. 50, no. 7, pp. 2901-2909, July 2012.

[5] F. Said and H. Johnsen, "Sea surface wind retrieval using both normalized radar cross section and polarization residual doppler frequency from TerraSAR-X data," in Geoscience and Remote Sensing Symposium (IGARSS), 2012 IEEE International, July 2012, pp. $2063-2066$.

[6] _ - "Ocean surface wind retrieval from dual-polarized sar data using the polarization residual doppler frequency," Geoscience and Remote Sensing, IEEE Transactions on, vol. 52, no. 7, pp. 3980-3990, Sep 2013.

[7] G. Engen, I. Friestad-Pedersen, H. Johnsen, and T. Elfouhaily, "Curvature effects in ocean surface scattering," IEEE Transactions on Antennas and Propagation, vol. 54, no. 5, pp. 1370-1379, May 2006.

[8] F. T. Ulaby, R. K. Moore, and A. K. Fung, Microwave Remote Sensing: Active and Passive Volume II: Radar Remote Sensing and Surface Scattering and Emission Theory, D. S. Simonett, Ed. Addison-Wesley, 1982. 
[9] P. Beckmann and A. Spizzichino, The Scattering of Electromagnetic Waves from Rough Surfaces. MacMillan, 1963, vol. 4.

[10] I. F. Pedersen, G. Engen, and H. Johnsen, "Polarization dependency in sea surface Doppler frequency and its application to envisat ASAR alt-pol data," in Envisat and ERS Symposium. Norut Information Technology, September 2004.

[11] F. Said, H. Johnsen, F. Nouguier, B. Chapron, and E. Geir, "Onto a skewness approach to the generalized curvature ocean surface scattering model," submitted in 2014 to Geoscience and Remote Sensing, IEEE Transactions on.

[12] G. Engen, P. W. Vachon, H. Johnsen, and F. W. Dobson, "Retrieval of ocean wave spectra and rar mtf's from dualpolarization SAR data," IEEE Transactions on Geoscience and Remote Sensing, vol. 38, no. 1, pp. 391-403, January 2000.

[13] C. Cox and W. Munk, "Measurement of the roughness of the sea surface from photographs of the sun's glitter," J. Opt. Soc. Am., vol. 44, no. 11, pp. 838-850, Nov 1954. [Online]. Available: http: //www.opticsinfobase.org/abstract.cfm?URI=josa-44-11-838

[14] F. M. Bréon and N. Henriot, "Spaceborne observations of ocean glint reflectance and modeling of wave slope distributions," Journal of Geophysical Research: Oceans, vol. 111, no. C6, pp. n/a-n/a, 2006. [Online]. Available: http://dx.doi.org/10.1029/2005JC003343

[15] H. Johnsen, G. Engen, and G. Guitton, "Sea-surface polarization ratio from envisat ASAR AP data," IEEE Transactions on Geoscience and Remote Sensing, vol. 46, no. 11, pp. 3637-3646, November 2008.

[16] V. Kudryavtsev, D. Hauser, G. Caudal, and B. Chapron, "A semiempirical model of the normalized radar cross-section of the sea surface," Journal of Geophysical Research, vol. 108, no. C3, p. 8054, 2003.

[17] M. Hansen, V. Kudryavtsev, B. Chapron, J. Johannessen, F. Collard, K.-F. Dagestad, and A. Mouche, "Simulation of radar backscatter and doppler shifts of wavecurrent interaction in the presence of strong tidal current," Remote Sensing of Environment, vol. 120, no. 0, pp. 113 - 122, 2012, the Sentinel Missions - New Opportunities for Science. [Online]. Available: http://www.sciencedirect.com/science/article/pii/S0034425712000703

TABLE I

STATISTICAL COMPARISON OF ESTIMATED $D_{c}$ WITH AND WITHOUT SKEWNESS AGAINST CDOP

$\left(\Delta D_{c}=\right.$ BIAS AND $\sigma_{D_{c}}=$ STANDARD DEVIATION $)-$ UNIT IN CM $/ \mathrm{S}$

\begin{tabular}{|c|c|c|c|c|c|c|c|c|c|c|c|c|c|c|c|c|c|c|}
\hline & \multicolumn{6}{|c|}{$u_{10}=5 \mathrm{~m} \cdot \mathrm{s}^{-1}$} & \multicolumn{6}{|c|}{$u_{10}=7 \mathrm{~m} \cdot \mathrm{s}^{-1}$} & \multicolumn{6}{|c|}{$u_{10}=10 \mathrm{~m} . \mathrm{s}^{-1}$} \\
\hline & \multicolumn{2}{|c|}{$\theta_{i}=26^{\circ}$} & \multicolumn{2}{|c|}{$\theta_{i}=32^{\circ}$} & \multicolumn{2}{|c|}{$\theta_{i}=40^{\circ}$} & \multicolumn{2}{|c|}{$\theta_{i}=26^{\circ}$} & \multicolumn{2}{|c|}{$\theta_{i}=32^{\circ}$} & \multicolumn{2}{|c|}{$\theta_{i}=40^{\circ}$} & \multicolumn{2}{|c|}{$\theta_{i}=26^{\circ}$} & \multicolumn{2}{|c|}{$\theta_{i}=32^{\circ}$} & \multicolumn{2}{|c|}{$\theta_{i}=40^{\circ}$} \\
\hline & hh & $\mathrm{vv}$ & hh & $\mathrm{vv}$ & hh & vv & hh & $\mathrm{vv}$ & hh & $\mathrm{vv}$ & hh & $\mathrm{vv}$ & hh & $\mathrm{vv}$ & hh & $\mathrm{vv}$ & hh & vv \\
\hline$\Delta D_{c}^{\text {noskew }}$ & -3.3 & -13.9 & -3.3 & -12.8 & -5.5 & -11.6 & -2.8 & -13.9 & -1.7 & -14.4 & -3.9 & -13.9 & -1.7 & -15.5 & -0.6 & -15 & -2.8 & -15 \\
\hline$\Delta D_{c}^{\text {skew }}$ & 5.5 & -5 & 5.5 & -6.1 & 1.7 & -7.8 & 16.1 & 3.3 & 14.4 & -2.8 & 7.8 & -7.8 & 29.4 & 13.3 & 30 & 7.2 & 21.1 & -2.2 \\
\hline$\sigma_{D_{c}}^{\text {noskew }}$ & 34.4 & 23.3 & 11.6 & 13.3 & 11.1 & 18.9 & 22.7 & 17.2 & 12.8 & 23.9 & 14.4 & 28.3 & 18.3 & 18.9 & 23.3 & 33.8 & 27.7 & 38.3 \\
\hline$\sigma_{D_{c} \text { skew }}$ & 9.4 & 9.4 & 6.7 & 15 & 12.8 & 19.4 & 21.6 & 18.3 & 10.5 & 18.3 & 10 & 23.3 & 32.7 & 27.7 & 22.7 & 22.2 & 11.1 & 23.9 \\
\hline
\end{tabular}


TABLE II

STATISTICAL COMPARISON OF ESTIMATED $D_{c}$ WITH AND WITHOUT WAVE BREAKING TERM AGAINST CDOP $\left(\Delta D_{c}=\right.$ BIAS AND $\sigma_{D_{c}}=$ STANDARD DEVIATION $)-$ UNIT IN CM/S

\begin{tabular}{|c|c|c|c|c|c|c|c|c|c|c|c|c|}
\hline & \multicolumn{6}{|c|}{$u_{10}=12 \mathrm{~m} \cdot \mathrm{s}^{-1}$} & \multicolumn{6}{|c|}{$u_{10}=15 \mathrm{~m} \cdot \mathrm{s}^{-1}$} \\
\hline & \multicolumn{2}{|c|}{$\theta_{i}=26^{\circ}$} & \multicolumn{2}{|c|}{$\theta_{i}=32^{\circ}$} & \multicolumn{2}{|c|}{$\theta_{i}=40^{\circ}$} & \multicolumn{2}{|c|}{$\theta_{i}=26^{\circ}$} & \multicolumn{2}{|c|}{$\theta_{i}=32^{\circ}$} & \multicolumn{2}{|c|}{$\theta_{i}=40^{\circ}$} \\
\hline & hh & $\mathrm{VV}$ & hh & $\mathrm{vV}$ & hh & $\mathrm{vV}$ & hh & vV & hh & $\mathrm{vv}$ & $\mathrm{hh}$ & $\mathrm{VV}$ \\
\hline$\Delta D_{c}^{n o b w}$ & 0.6 & -13.9 & 1.7 & -12.8 & -1.1 & -13.3 & 2.2 & -8.9 & 2.8 & -8.3 & -2.2 & -10 \\
\hline$\Delta D_{c}^{b w}$ & -1.1 & -14.4 & 0.6 & -12.2 & 0 & -10 & -0.6 & -10.5 & 0.6 & -7.8 & -2.2 & -6.1 \\
\hline$\sigma_{D_{c}}^{n o b w}$ & 38.8 & 26.1 & 14.4 & 19.4 & 16.6 & 30 & 49.9 & 34.9 & 18.9 & 18.3 & 19.4 & 33.3 \\
\hline$\sigma_{D_{c}}^{b w}$ & 28.3 & 22.2 & 12.2 & 13.9 & 11.6 & 19.4 & 29.4 & 25 & 13.9 & 13.9 & 15 & 20 \\
\hline
\end{tabular}

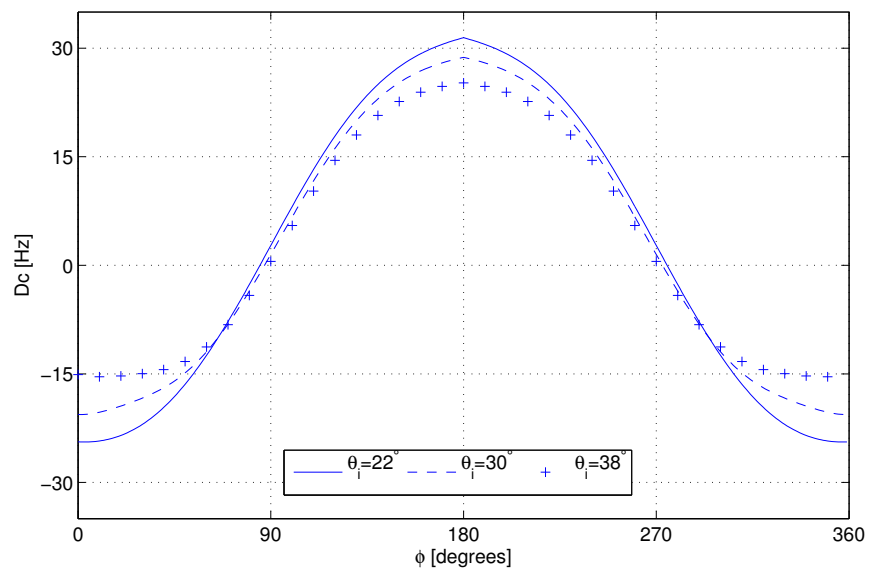

Fig. 1. Plots of the $D_{c}$ in terms of the wind direction, using CDOP for a $v v$ polarization state and a $10 \mathrm{~m} / \mathrm{s}$ wind speed. A 0 degree wind direction corresponds to down-wind. 

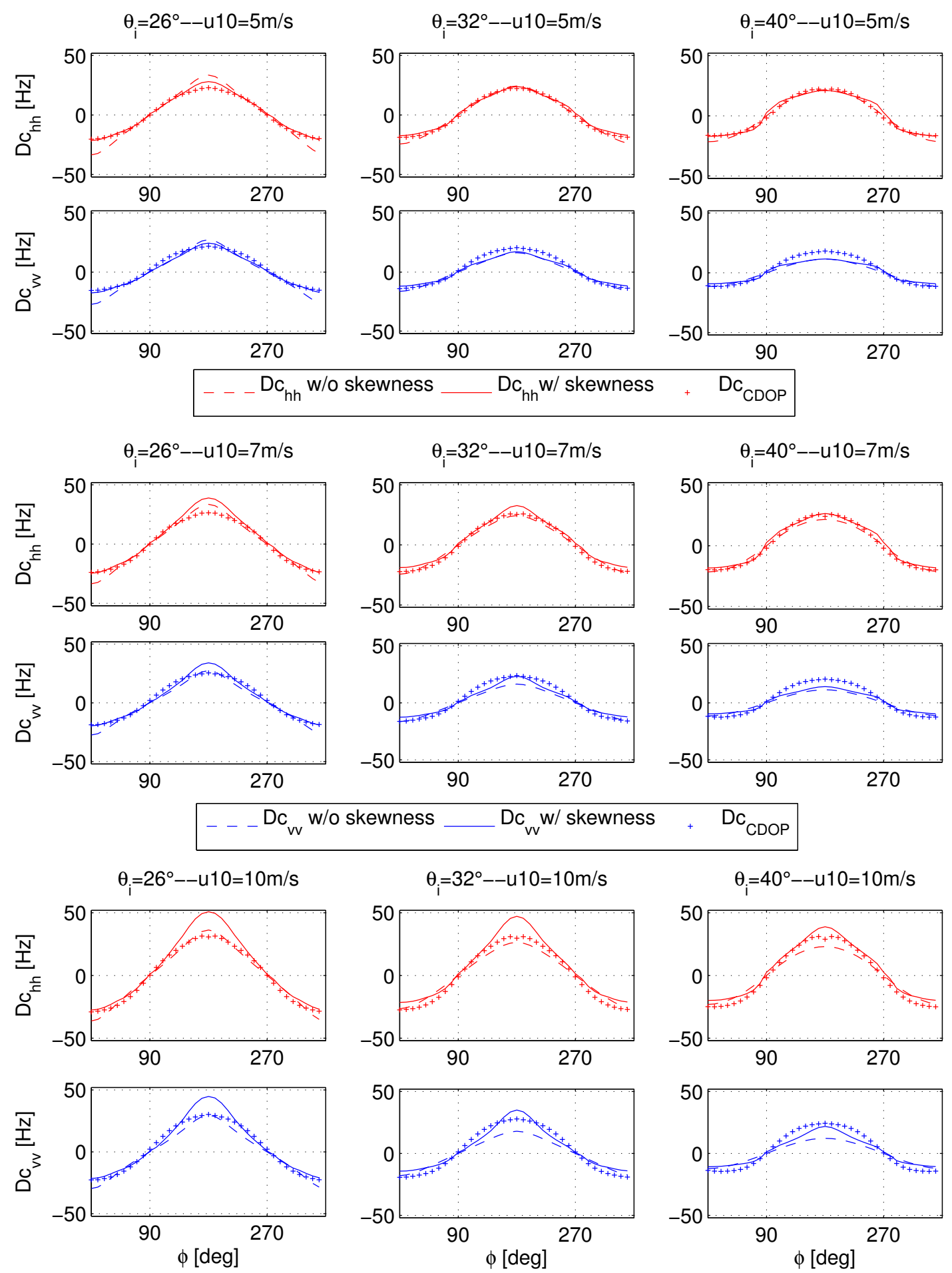

Fig. 2. Comparison of the GCM $D_{c}$ model with and without the skewness related phase parameter. It is compared against CDOP for both the $h h$ and $v v$ polarizations. Analysis is done for $\mathrm{u}_{10}=5,7$, and $10 \mathrm{~m} / \mathrm{s}$, and three different incidence angles (26, 32, and 40 degrees). The wave breaking component from section II-C is not included. A 0 degree wind direction corresponds to down-wind. 

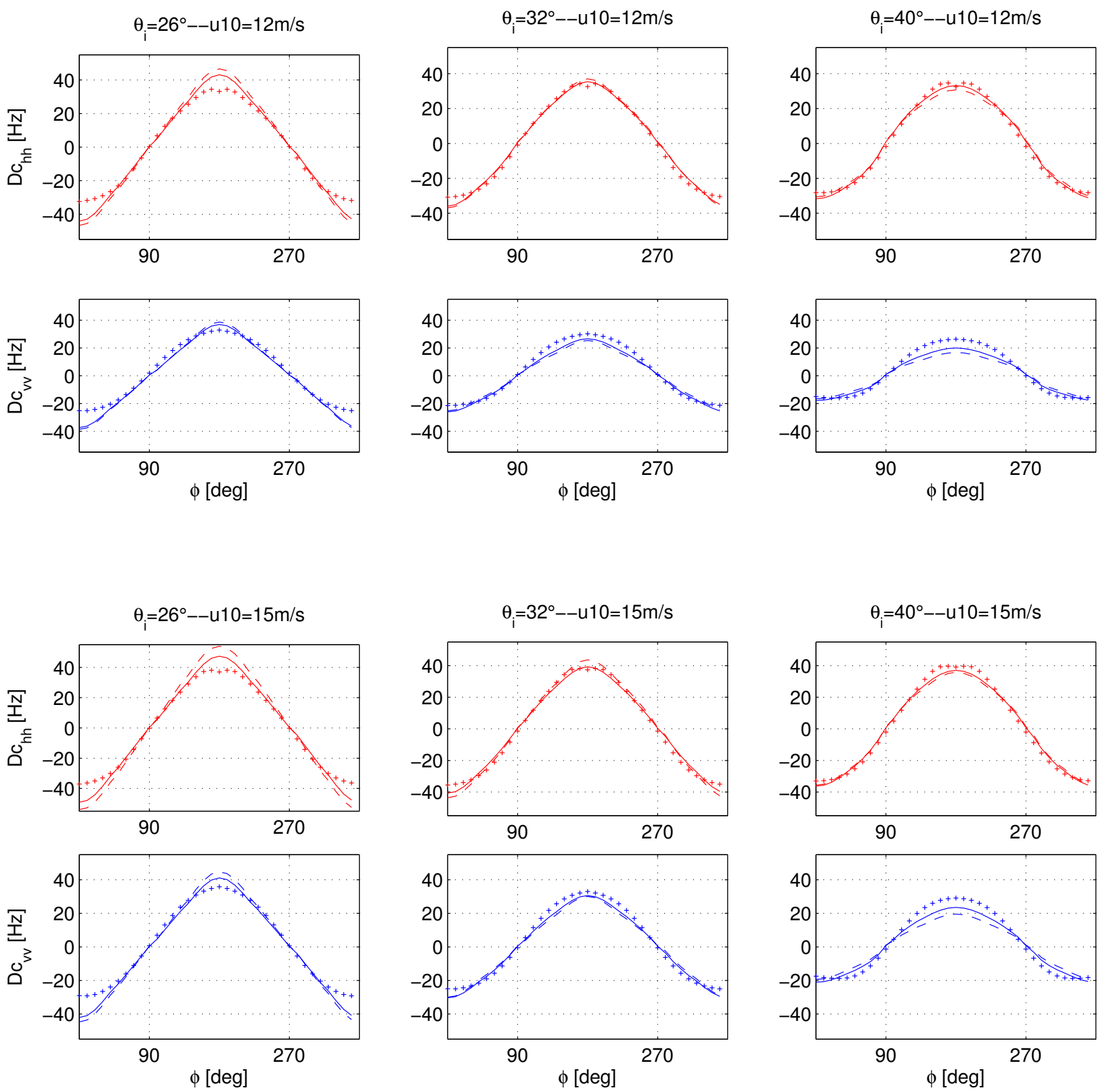

$-\ldots-D c_{h h} w / 0 b w \ldots D c_{h h} w / b w+D c_{C D O P}+-D_{v v} w / o b w \ldots D c_{v v} w / b w+D c_{C D O P}$

Fig. 3. Comparison of the GCM $D_{c}$ model with and without the wave breaking component. It is compared against CDOP for both the $h h$ and $v v$ polarizations. Analysis is done for $\mathrm{u}_{10}=12$ and $15 \mathrm{~m} / \mathrm{s}$, and three different incidence angles (26, 32, and 40 degrees). Note that the skewness phase related component is not included. A 0 degree wind direction corresponds to down-wind. 